\title{
Atlanto-axial rotatory subluxations in postmortem CT: radiologists be aware of a common pitfall
}

\author{
Anders Persson, Josef Falk, Johan Berge and Christian Jackowski
}

\section{Linköping University Post Print}

N.B.: When citing this work, cite the original article.

Original Publication:

Anders Persson, Josef Falk, Johan Berge and Christian Jackowski, Atlanto-axial rotatory subluxations in postmortem CT: radiologists be aware of a common pitfall, 2013, Forensic Science International, (225), 1-3, 9-14.

http://dx.doi.org/10.1016/j.forsciint.2013.01.001

Copyright: Elsevier http://www.elsevier.com/

Postprint available at: Linköping University Electronic Press http://urn.kb.se/resolve?urn=urn:nbn:se:liu:diva-90221 
Elsevier Editorial System(tm) for Forensic Science International Manuscript Draft

Manuscript Number: FSI-D-12-00089R1

Title: Atlanto-Axial Rotatory Subluxations in Postmortem CT: Radiologists Be Aware of a common Pitfall.

Article Type: Special Issue: Postmortem Imaging

Section/Category: Questioned Documents and Physical Science

Keywords: Computed tomography * postmortem spine imaging * atlanto-axial rotatory subluxations

Corresponding Author: Director CMIV, Assoc. Professor Anders Persson, MD, PhD

Corresponding Author's Institution: Institution for medical health

First Author: Anders Persson, MD. PhD

Order of Authors: Anders Persson, MD. PhD; Josef Falk, MD.; Johan Berge, MD; Christian Jackowski, MD

Manuscript Region of Origin: SWEDEN

Abstract: The aim of the present study was to determine the frequency of atlanto-axial rotatory subluxations (AARS) in multi detector computed tomography (MDCT) performed on human corpses for forensic purposes and to investigate whether these are a physiological postmortem finding or indicate a trauma to the neck region.

80 forensic cases examined with MDCT from November 2003 to March 2007 were included in the study. The study was approved by the regional ethics committee. For each case volumes were rendered and investigated with reference to suspected AARS and any other anomalies of the head and neck region. The rotation of the head as well as in the atlanto-axial joint were measured and occurring AARS were judged according Fielding`s classification. The finding of AARS was correlated to case criteria such as postmortem head rotation, sex, age, cause of death, time since death and further autopsy results. Statistical analysis was performed using Fisher's exact test, Wilcoxon`s rank sums test and Chisquare test with Pearson approximation.

$70 \%(n=56)$ of the cases included in the study presented with an AARS. A strong correlation $(\mathrm{p}<.0001)$ between suspected AARS and postmortem head rotation was found. Two cases presented with an atlanto-axial rotation greater than the head rotation. One showed an undiscovered lateral dislocation of the atlas, and one an unfused atlas-ring. There was no correlation to any further investigated case criteria. Ipsilateral AARS with head rotation alone does not indicate trauma to the neck. PmCT can substantially support forensic examinations of the skeleton, especially in body regions, which are elaborate to access at autopsy, such as the cervical spine. Isolated AARS (Fielding type I) on pmCT is usually a normal finding associated with ipsilateral head rotation.

Suggested Reviewers: 


\title{
Atlanto-Axial Rotatory Subluxations in Postmortem CT: Radiologists Be Aware of a common Pitfall.
}

\author{
A. Persson ${ }^{\mathrm{a}}, \mathrm{J}_{\text {. Falk }}$, J. Berge ${ }^{\mathrm{b}}$, C. Jackowski ${ }^{\mathrm{c}}$ \\ ${ }^{a}$ Center for Medical Image Science and Visualization (CMIV), University of Linköping, 58185 \\ Linköping, Sweden \\ ${ }^{\mathrm{b}}$ Department of Forensic Medicine (RMV), Artillerigatan 12, 58758 Linköping, Sweden \\ ${ }^{\mathrm{c}}$ Center for Forensic Imaging Institute of Forensic Medicine University of Bern Bühlstr. 20 \\ CH 3012 Bern Switzerland
}

Address correspondence to:

Anders Persson MD. PhD. Professor

Center for Medical Image Science and Visualization (CMIV),

University of Linköping, 58185 Linköping, Sweden

Phone: +46-10-103 8906

Fax +46-10-103 7020

Email: anders.persson@cmiv.liu.se 
*Manuscript (without author identifiers)

Click here to view linked References

\section{Atlanto-Axial Rotatory Subluxations in Postmortem CT:}

Radiologists Be Aware of a common Pitfall. 


\section{ABSTRACT}

The aim of the present study was to determine the frequency of atlanto-axial rotatory subluxations (AARS) in multi detector computed tomography (MDCT) performed on human corpses for forensic purposes and to investigate whether these are a physiological postmortem finding or indicate a trauma to the neck region.

80 forensic cases examined with MDCT from November 2003 to March 2007 were included in the study. The study was approved by the regional ethics committee. For each case volumes were rendered and investigated with reference to suspected AARS and any other anomalies of the head and neck region. The rotation of the head as well as in the atlanto-axial joint were measured and occurring AARS were judged according Fielding`s classification. The finding of AARS was correlated to case criteria such as postmortem head rotation, sex, age, cause of death, time since death and further autopsy results. Statistical analysis was performed using Fisher's exact test, Wilcoxon`s rank sums test and Chi-square test with Pearson approximation.

$70 \%(\mathrm{n}=56)$ of the cases included in the study presented with an AARS. A strong correlation $(\mathrm{p}<.0001)$ between suspected AARS and postmortem head rotation was found. Two cases presented with an atlanto-axial rotation greater than the head rotation. One showed an undiscovered lateral dislocation of the atlas, and one an unfused atlas-ring. There was no correlation to any further investigated case criteria. Ipsilateral AARS with head rotation alone does not indicate trauma to the neck.

PmCT can substantially support forensic examinations of the skeleton, especially in body regions, which are elaborate to access at autopsy, such as the cervical spine. Isolated AARS (Fielding type I) on pmCT is usually a normal finding associated with ipsilateral head rotation.

Keywords: Computed tomography $\bullet$ postmortem spine imaging • atlanto-axial rotatory subluxations 


\section{Introduction}

In recent years postmortem examinations are increasingly supported by cross sectional imaging. Predominantly CT is used to enhance the diagnostic possibilities in foreign body detection, skeletal findings and gaseous pathologies [1-9]. Since 2003, postmortem CT (pmCT) is added to forensic examinations of deceased at the local Department of Forensic Medicine, which is performed in close collaboration with the Center for Medical Science and Visualization (CMIV) at Linköping University [10]. Prior to traditional autopsy, the human corpses are examined at CMIV using multi detector computed tomography (MDCT), which has been implemented on a routine basis to complement traditional autopsy, especially when skeletal or gaseous findings are of importance in certain cases. One advantage is that the body and evidence attached to it are documented as they were delivered to the institution before the staff has opened the body bag or even touched the corpse. Thus, a full set of images of the body and its internal organs is acquired without the risk of compromising any evidence on the body. The image data can also be stored indefinitely and later revised at any time to answer newly arisen questions or to serve as an independent base for second opinions.

After initiation of the pmCT technique in the clinic several of the examined cases presented with cervical spine findings that were interpreted as atlanto-axial rotatory subluxations (AARS) by the reading radiologists and reported as a possible cause of death (Figure 1). However, this finding could not be confirmed by the forensic pathologists at autopsy. Most of the corpses neither showed other signs of pathologic changes to the neck region, nor any information was found in the police reports suggesting violence against the neck, which could provide a comprehensible explanation for the phenomenon. Thereby, a need for a study arose, which investigates the frequency of AARS in pmCT and correlates it to factors that might contribute to its occurrence. 
The rotation of the human cervical spine has been studied using different approaches and techniques, both in vivo and in vitro [11-19]. Researchers report a mean maximum range of rotation for the head relative to the body of $60-80^{\circ}$ to both sides $[12-14,19,20] .50-60 \%$ of this movement, or a mean of $30-45^{\circ}$, takes place in the atlanto-axial joint (C1-C2) [12-16,20], and the rest is distributed at the lower levels of the cervical column (C2-T1), C0-C1 having only a minor contribution [12-14,16,20]. For some individuals, a normal maximum rotation in the atlanto-axial joint of over $50^{\circ}$ has been reported[11,13], and Dvorak and co-workers suggest that hypermobility or instability of the occipito-atlanto-axial complex should be suspected if the maximum rotation exceeds $56^{\circ}$ at the C1-C2-segment [13].

AARS may be seen in torticollis or its prolonged state rotatory fixation, conditions that may have a spontaneous onset or follow infections of the upper respiratory tract, neck trauma or surgical operations in the face and neck region [20-22]. Fielding and Hawkins classified such fixations into four different types, based on the radiological appearance of the atlantoaxial complex: Type I (rotatory fixation without displacement of the atlas), and types II-IV (rotatory fixations combined with different degrees of anterior and posterior displacements of the atlas) [21]. Atlanto-axial fixation may occur with subluxation or dislocation of the atlantoaxial complex, but it may also occur within what is believed to be the vertebras normal range of motion [18,21]. According to Kowalski et al, it is virtually impossible to distinguish radiographically the physiological rotation between the atlas and the axis that occurs in the normal rotation of the head from the pathological subluxations in torticollis and atlanto-axial fixation [15].

To investigate and describe the finding of AARS in deceased gained in importance as it might represent a typical pitfall in post-mortem CT data reading. Systematic false positive diagnosis for AARS would unnecessarily compromise the reliability of CT in forensic examinations, especially when the CT data enter the courtroom as evidence. The aim of the 
present study was to determine the frequency of suspected AARS in pmCT data sets and to discuss possible mechanisms of its aetiology.

\section{Materials and methods}

\subsection{Data acquisition}

From November 2003 to March 2007, 80 forensic cases (51 males, 31 females; mean age 37.1 years, range 2 months -87 years, $\mathrm{SD}=24.8$ ) underwent pmCT examinations. The regional ethics committee approved the study.

\section{Scanning interval 1}

From November 2003 to September 2006, 63 of the bodies included in the study were scanned by means of a 16 detector row CT scanner (Somatom Sensation 16, Siemens Medical Systems, Forchheim, Germany) with $16 \times 1 \mathrm{~mm}$ collimation, adaptive pitch, $200 \mathrm{mAs}$ and $120 \mathrm{kV}$.

\section{Scanning interval 2}

From October 2006 to March 2007, 17 pmCT examinations were performed using a Dual source 2 x 64 detector CT scanner (Somatom Definition, Siemens Medical Systems, Forchheim, Germany) with $2 \times 64 \times 0.6 \mathrm{~mm}$ collimation, adaptive pitch, dose modulation with reference mAs of 200 and $120 \mathrm{kV}$.

Full body scans were reconstructed with a voxel size of to $0.7 \times 0.7 \times 0.7 \mathrm{~mm}$. Specific scan protocols were added depending on suspected injuries to the body to be further examined. For head- and neck examinations, a field of view (FOV) of an approximate mean of $20 \mathrm{~cm}$ was used with a voxel size down to $0.4 \times 0.4 \times 0.4 \mathrm{~mm}$. 


\subsection{Image Display and Analysis}

For image display and image post-processing, a 3D Workstation (Multi modality workstation with 3D software and Inspace interactive 3D Viewer, Siemens Medical Solutions Computed Tomography, Forchheim, Germany) equipped with a real-time volume rendering graphic accelerator card was used.

Volumes were rendered visualizing the bony parts of the head, neck and shoulders. The volumes were oriented in a caudal view, and a software-provided measure grid was adjusted parallel to the axis of both shoulders (Figure 2a). Using a software-provided anglemeasurement tool and an axial clip-plane applied at different levels of the volume, the rotation of the head relative to the shoulders was measured. The nasal septum and the foramen magnum were used as reference points on the grid (Figures $2 b$ and $2 c$ ). The same procedure was repeated at the level of $\mathrm{C} 1-\mathrm{C} 2$, whereas the grid was instead adjusted to the axis (Figure $3 a$ ), and the rotation of the atlas relative to the axis was measured using the foramina transversaria and the transverse processus of the atlas as reference points (Figures $3 \mathrm{~b}$ and $3 \mathrm{c}$ ).

The presence and type of AARS corresponding to Fielding et al.'s classification system [21] was noted, as were any other findings such as dislocations of the vertebras, fractures and soft tissue injuries of the head and neck. The factors that may make it an abnormal finding are Fielding types II - V (various displacements of the atlas in relation to the dens), finding of blood around the spinal cord, degree of head rotation > AARS and other bone injury e.g. fractures in the cervical spine.

The evaluation of all examinations was first carried out individually (JF and AP). The readers then solved minor disagreements in a consensus session. 


\subsection{Forensic documentation}

For each of the cases, information about the following variables was collected from the forensic documentation: Age, sex, height, weight and Body Mass Index (BMI), cause of death, head and neck injuries, alcohol-levels in blood and urine, results from the drugscreening, presence of rigor mortis in the limbs and the neck at the time of the autopsy, the forensic pathologists notion on whether the neck felt stable or not, skeletal injury, soft tissue damage, ligament damage and other detectable changes in the cervical spine region, time passed from the time of death to the time of the CT-examination, place of finding (outdoors, indoors or water), and body and head positions at the time of the finding (lying supine, prone or on one side, sitting, head rotated or not, direction of head rotation.

\subsection{Statistical Analysis}

Statistical analysis was performed using SAS software (JMP version 7.0.1, SAS Institute Inc., Cary, NC, USA). The group that radiographically presented with what was interpreted as an AARS was compared to the group in which the phenomenon was absent, to find any differences regarding possible influences of any of the factors on which information was collected. Fisher's exact test, Wilcoxon's rank sums test and Chi-square test with Pearson approximation were used to compare bivariate, continuous and nominal data, respectively. The correlation between atlanto-axial rotation and head rotation was investigated using both Fisher's exact test and a correlation analysis with linear fit. $\mathrm{P}<.05$ was considered to indicate significance.

\section{Results}

From all forensic cases examined with volume rendered CT $70 \%$ were judged to have a suspected AARS (N=56), all corresponding to type I of Fielding's classification [21]. In this 
group, the rotation of the atlas on the axis ranged from $4^{\circ}$ to $47^{\circ}$ (left or right), with a mean value of $20^{\circ}(\mathrm{SD}=16.2)$.

There was a strong correlation $(\mathrm{p}<.0001)$ between the presence of suspected AARS and postmortem left or right rotation of the head (Table 1). Figure 4 shows that AARS typically appears to begin at about $10^{\circ}-20^{\circ}$ of head rotation, and is then proportionally increasing with increased head rotation. There was a correlation of borderline significance $(p=.053)$ between time passed from the time of death to the time of the CT-examination and the presence of suspected AARS (Table 2). No significant correlations between the presences of suspected AARS and any of the other factors that we collected information about was found (Tables 14).

In 19 cases, the forensic pathologist noted that the "cervical spine feels stable" in the autopsy report. 13 of these presented radiographically with a suspected AARS (atlanto-axial rotation ranging from $16-47^{\circ}$, both left and right). Two cases were noted in the autopsy report to have a cervical spine that felt unstable, and although both had injuries to the cervical vertebrae, only one presented radiographically with a suspected AARS $\left(42^{\circ}\right)$.

Two cases presented with a $\mathrm{C} 1-\mathrm{C} 2$ rotation substantially greater than the rotation of the head $\left(22^{\circ}\right.$ vs. $11^{\circ}$ and $18^{\circ}$ vs. $8^{\circ}$, respectively). Both showed further cervical spine abnormalities such as a lateral dislocation of the atlas or a right lateral cleft of the posterior arch of the atlas (Fig 5).

Too few observations were available to give a reliable statistic result regarding the drugscreening results (except for alcohol-levels in blood and urine), place of finding, body and head positions and presence of rigor mortis.

\section{Discussion}

The present study was conducted after several false positive radiological diagnoses of 
AARS on postmortem CT data occurred at our department.

Our study results suggest that what is clinically known as AARS can be frequently found in CT-examinations of human corpses. It is not a sign of any pathologic alteration or trauma to the neck, but a normal finding of a physiological postmortem head rotation as long as the AARS is not exceeding the rotation of the head, occurs ipsilateral and further signs of trauma are lacking. This is important to acknowledge as it presents a potential pitfall: In the clinic the radiological presence of an AARS indicates a pathologic state, but not necessarily so in human cadavers.

On the living patient a CT-scan is conducted with the patient lying with the head in a neutral position. Therefore, when a patient presents with an AARS, this indicates a pathologic state such as torticollis or rotatory fixation of the neck. Forensic corpses undergoing CT-scans are usually examined at the same position as they were wrapped at the death scene. This can be almost every imaginable position partly far differing from known patient scanning positions. Depending on the time since death it would even be impossible to get the corpse in a "correct" scanning position due to heavily developed rigor mortis. Therefore, for this heterogeneous group it can be expected that several individuals have their heads rotated and that the finding of AARS represents the physiological participation of the C1-C2 joint.

Two cases presented with a $\mathrm{C} 1-\mathrm{C} 2$ rotation greater than the rotation of the head, and both showed pathological changes of the occipito-atlanto-axial complex, anomalies that had until then gone undiscovered. One exhibited a lateral dislocation of the atlas (Figure 5a). The case was a male in his mid teens. The cause of death was established to be fatal internal hemorrhage following a knife stab wound to the trunk, and no information was found in the forensic documentation suggesting any significant violence against the neck. The second one presented with a cleft of the posterior arch of the atlas (Figure 5b). The cause of death was established to be lung insufficiency following chest trauma in combination with alcohol 
intoxication. In this case there was also a notion on "signs of blunt violence against the neck" in the autopsy report. However, the cleft of the posterior arch of the atlas was interpreted to be a congenital anomaly, as described among others by Gehweiler et al. [23]. A single fracture of the atlas is very unlikely and described as "exceedingly rare" [24]. On the other hand, it would be plausible that the absence of a fully developed posterior arch also indicates altered ligamentous and/or other anatomical conditions, predisposing a displacement of the atlas.

However, Yen et al. reported a case of a AARS $\left(31^{\circ}\right)$ in a poly trauma victim combined with medullary transection diagnosed on postmortem CT and MRI [25]. Therefore, we suggest that readers make it routine to carefully examine the configuration of the occipitoatlanto-axial complex in the radiological examination, as well as to compare the rotation of the atlanto-axial joint to the rotation of the head, as these easy measures can rule out or bring attention to pathological conditions in this important area. We also suggest extra attention being paid if a rotation between C1-C2 is found that approaches or exceeds the $56^{\circ}$ described in the literature to be the lower limit for suspected hypermobility or instability [13].

There was a correlation of borderline significance $(\mathrm{p}=.053)$ between time passed from the time of death to the time of the CT-examination and the presence of suspected AARS (Table 2). However, this correlation analysis was based on only 32 observations, and it is possible that future studies based on more observations might result in a weaker correlation.

As a study limitation it needs to be mentioned that it was not discriminated between adult and child cases. It is possible that the physiological post mortem state regarding the neck region is not completely comparable as the vertebral bodies are not fully developed in infants. Furthermore, possible influences on the measured values for head and atlanto-axial rotation from the head being abducted or flexed/extended relative to its neutral position have not be taken into account. As a head rotation is mostly not only associated with horizontal rotation at different levels of the spine, but also with coupled movements of vertebras in the other planes 
$[14,19,20]$ it might be possible that rotation combined with abduction and/or flexion/extension of the head have altered the degree of rotation in the atlanto-axial joint. However, the purpose of this study was to examine the horizontal rotation within the atlas-axis joint and the influence of a possible variation in head adduction and flexion/extension was expected to be relatively negligible.

For some of the factors that were investigated for correlation with suspected AARS, the results are based on relatively few observations. Future studies including a greater number of observations may confirm our results and may also describe the significance, if any, of congenital atlas malformations in suspected AARS and other pathological changes of the cervical region found in CT-examinations of forensic cases.

\section{Conclusion}

In conclusion, what looks like AARS in corpses undergoing postmortem CT examinations appears to be the normal atlanto-axial rotation associated with an ipsilateral head rotation, and has usually not to be considered any pathological meaning. Close attention should be paid to the occipito-atlanto-axial complex in postmortem CT data reading, as to not misinterpret or overlook findings and anomalies in this vitally important region 


\section{References}

[1] A. D. Levy, R. M. Abbott, C. T. Mallak, J. M. Getz, H. T. Harcke, H. R. Champion, L. A. Pearse. Virtual autopsy: preliminary experience in high-velocity gunshot wound victims. Radiology 240 (2006) 522-528.

[2] Y. Oyake, T. Aoki, S. Shiotani, M. Kohno, N. Ohashi, H. Akutsu, K. Yamazaki. Postmortem computed tomography for detecting causes of sudden death in infants and children: retrospective review of cases. Radiat Med 24 (2006) 493-502.

[3] A. D. Levy, H. T. Harcke, J. M. Getz, C. T. Mallak, J. L. Caruso, L. Pearse, A. A. Frazier, J. R. Galvin. Virtual autopsy: two- and three-dimensional multidetector CT findings in drowning with autopsy comparison. Radiology 243 (2007) 862-868.

[4] C. Jackowski, M. Thali, M. Sonnenschein, E. Aghayev, K. Yen, R. Dirnhofer, P. Vock. Visualization and quantification of air embolism structure by processing postmortem MSCT data. J Forensic Sci 49 (2004) 1339-1342.

[5] S. Shiotani, M. Kohno, N. Ohashi, K. Yamazaki, H. Nakayama, K. Watanabe, Y. Oyake, Y. Itai. Non-traumatic postmortem computed tomographic (PMCT) findings of the lung. Forensic Sci Int 139 (2004) 39-48.

[6] R. Dirnhofer, C. Jackowski, P. Vock, K. Potter, M. J. Thali. VIRTOPSY: minimally invasive, imaging-guided virtual autopsy. Radiographics 26 (2006) 1305-1333.

[7] C. Jackowski, M. J. Thali, U. Buck, E. Aghayev, M. Sonnenschein, K. Yen, R. Dirnhofer, P. Vock. Noninvasive estimation of organ weights by postmortem magnetic resonance imaging and multislice computed tomography. Invest Radiol 41 (2006) 572578.

[8] E. Aghayev, M. Sonnenschein, C. Jackowski, M. Thali, U. Buck, K. Yen, S. Bolliger, R. Dirnhofer, P. Vock. Postmortem radiology of fatal hemorrhage: measurements of cross-sectional areas of major blood vessels and volumes of aorta and spleen on MDCT and volumes of heart chambers on MRI. AJR Am J Roentgenol 187 (2006) 209-215.

[9] C. Jackowski, M. Sonnenschein, M. J. Thali, E. Aghayev, K. Yen, R. Dirnhofer, P. Vock. Intrahepatic gas at postmortem computed tomography: forensic experience as a potential guide for in vivo trauma imaging. J Trauma 62 (2007) 979-988.

[10] P. Ljung, C. Winskog, A. Persson, C. Lundstrom, A. Ynnerman. Full body virtual autopsies using a state-of-the-art volume rendering pipeline. IEEE Trans Vis Comput Graph 12 (2006) 869-876.

[11] S. WERNE. Studies in spontaneous atlas dislocation. Acta Orthop Scand Suppl 23 (1957) 1-150.

[12] J. Dvorak, M. Panjabi, M. Gerber, W. Wichmann. CT-functional diagnostics of the rotatory instability of upper cervical spine. 1 . An experimental study on cadavers. Spine 12 (1987) 197-205. 
[13] J. Dvorak, J. Hayek, R. Zehnder. CT-functional diagnostics of the rotatory instability of the upper cervical spine. Part 2. An evaluation on healthy adults and patients with suspected instability. Spine 12 (1987) 726-731.

[14] L. Penning, J. T. Wilmink. Rotation of the cervical spine. A CT study in normal subjects. Spine 12 (1987) 732-738.

[15] H. M. Kowalski, W. A. Cohen, P. Cooper, J. H. Wisoff. Pitfalls in the CT diagnosis of atlantoaxial rotary subluxation. AJR Am J Roentgenol 149 (1987) 595-600.

[16] J. L. Dumas, R. M. Sainte, P. Dreyfus, D. Goldlust, J. P. Chevrel. Rotation of the cervical spinal column: a computed tomography in vivo study. Surg Radiol Anat 15 (1993) 333-339.

[17] J. L. Dumas, P. Thoreux, P. Attali, D. Goldlust, J. P. Chevrel. Three-dimensional CT analysis of atlantoaxial rotation: results in the normal subject. Surg Radiol Anat 16 (1994) 199-204.

[18] N. Schwarz, M. Lenz, A. Berzlanovich, W. Smetka. [Atlanto-axial rotation and distance in small children. A postmortem study]. Unfallchirurg 103 (2000) 656-661.

[19] E. M. Malmstrom, M. Karlberg, P. A. Fransson, A. Melander, M. Magnusson. Primary and coupled cervical movements: the effect of age, gender, and body mass index. A 3 dimensional movement analysis of a population without symptoms of neck disorders. Spine 31 (2006) E44-E50.

[20] White AA, Panjabi MM. Clinical Biomechanics of the Spine. 2nd ed. Lippincott Williams \& Wilkins, 1990.

[21] J. W. Fielding, R. J. Hawkins. Atlanto-axial rotatory fixation. (Fixed rotatory subluxation of the atlanto-axial joint). J Bone Joint Surg Am 59 (1977) 37-44.

[22] T. B. Crook, C. A. Eynon. Traumatic atlantoaxial rotatory subluxation. Emerg Med J 22 (2005) 671-672.

[23] J. A. Gehweiler, Jr., R. H. Daffner, L. Roberts, Jr. Malformations of the atlas vertebra simulating the Jefferson fracture. AJR Am J Roentgenol 140 (1983) 1083-1086.

[24] T. Inaoka, K. Ohashi, G. Y. El-Khoury. A single fracture in the atlas ring: report of two cases and a review of the literature. Emerg Radiol 14 (2007) 191-194.

[25] K. Yen, M. Sonnenschein, M. J. Thali, C. Ozdoba, J. Weis, K. Zwygart, E. Aghayev, C. Jackowski, R. Dirnhofer. Postmortem multislice computed tomography and magnetic resonance imaging of odontoid fractures, atlantoaxial distractions and ascending medullary edema. Int J Legal Med 119 (2005) 129-136. 


\section{Acknowledgments}

We would like to thank Elisabeth Wåhlin, Peggy Florhed-Hermansson and Nobar Ashjian (autopsy technicians) for their logistic support and Petter Quick as well as Agneta Wistingsgård (radiological technicians) for their experienced assistance in data acquisition and finally Lennart Rammer, Erik Edston, Håkan Bengtsson, Henrik Druid, Calle Winskog, Fredrik Bäckström and Jonas Lindström (forensic pathologists) performing most of the autopsies. We are also grateful to Örjan Smedby, M.D. for his support in statistical analysis. We are grateful for funding from National Board of Forensic Medicine. 


\section{Legends}

Fig. 1. Volume rendered cranial pmCT data presenting with a suspected atlanto-axial rotatory subluxation (AARS). Note the position of the right lateral mass of the first cervical vertebra (arrow) compared to the column of right lateral masses of the lower vertebras.

Fig. 2. Measurement of head rotation relative to the body on caudal views on volume rendered pmCT data. (a) A measure grid was adjusted parallel/perpendicular to the shoulders. (b) An axial clip plane was applied at a level through the nasal septum. (c) The nasal septum and the foramen magnum were used as reference points on the grid to measure the angle of head rotation (example case $\left.-25.0^{\circ}\right)$.

Fig. 3. Measurement of atlanto-axial rotation on the same volume as in Figure 2. Cranial view. (a) A horizontal clip plane was applied at a level slightly cranial to the axis (C2), and a measure grid adjusted parallel/perpendicular to the axis. (b) The clip plane was then raised to a level slightly cranial to the atlas (C1). (c) The foramina transversaria and the transverse processūs of the atlas were used as reference points on the grid to measure the angle of the atlanto-axial rotation (example case $-25.9^{\circ}$ ).

Fig. 4. Bivariate fit of $\mathrm{C} 1-\mathrm{C} 2$ rotation by head rotation relative to the body (ipsilateral left or right). $\mathrm{P}<.0001$ 
Fig. 5. Pathological findings in the $\mathrm{C} 1-\mathrm{C} 2$ region. (a) Cranial view with axial clip plane at a level slightly cranial to the atlas demonstrates a laterally dislocated atlas. Note the different distance between the dens and the left and right lateral masses of atlas (arrows). (b) Dorsal view on a non-closed posterior arch of the atlas (arrow).

\section{Table 1}

Correlation between head rotation, sex and forensic pathologist's notion on the stability of the neck to the occurrence of suspected AARS.

P-value for Fisher's exact test (2-tailed).

\section{Table 2}

Correlation between continuous variables and suspected AARS.

P-values for Wilcoxon/Kruskal-Wallis 2-sample rank sums test. BMI=Body Mass Index.

\section{Table 3}


P-values for Fisher's exact test (2-tailed). Note that several of the 80 cases were designated two or more causes of death (combined cause of death). CO poisoning - carbon monoxide poisoning. SIDS - sudden infant death syndrome.

\section{Table 4.}

\section{Correlation of AARS to autopsy results on head and neck}

P-values for Fisher's exact test (2-tailed).

* Radiologically or in the traditional autopsy visible injuries such as knife wounds, possibly damaging ligaments of the spine. 


\section{Figure 1}

Click here to download high resolution image

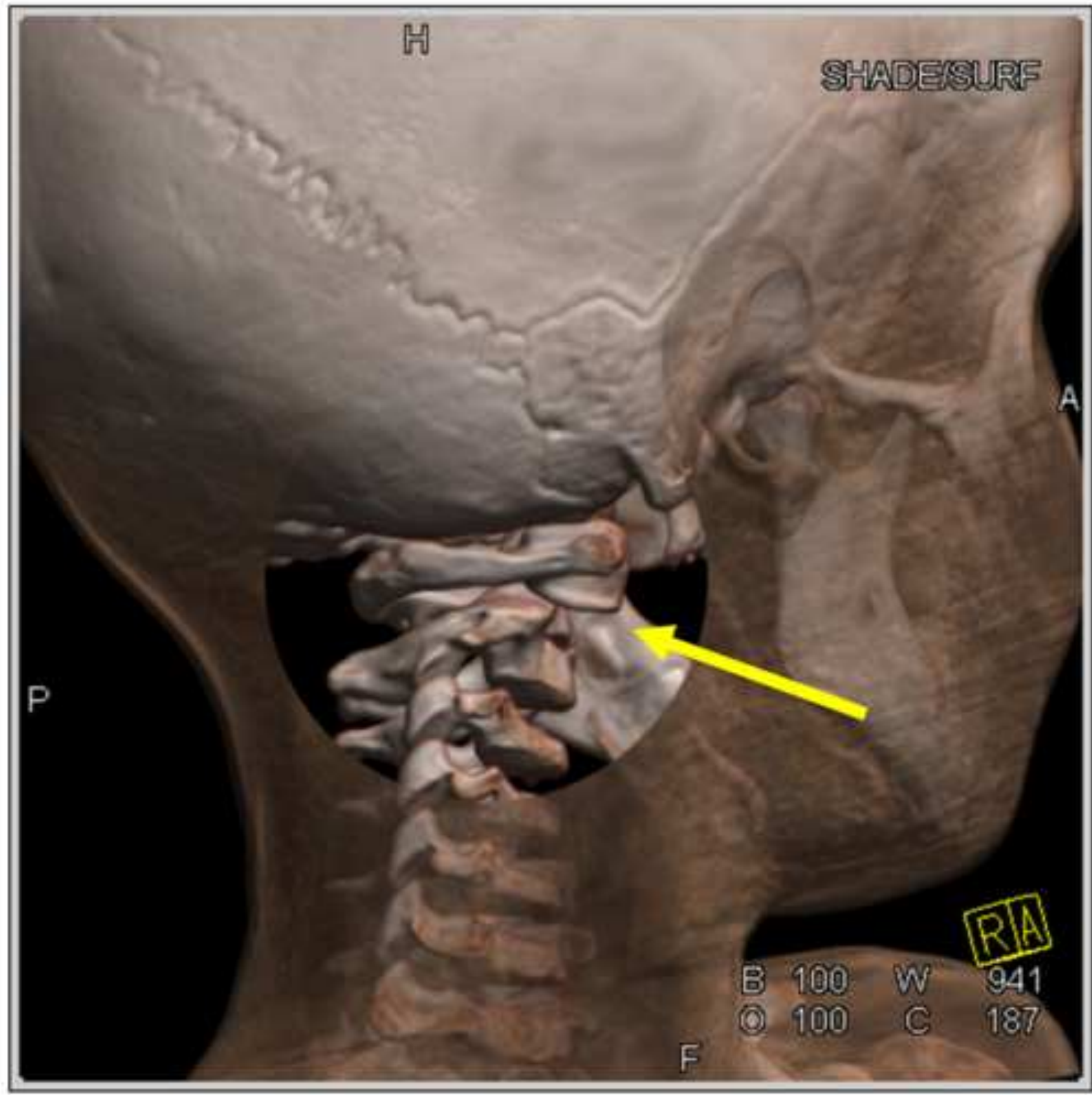

Figure 
Click here to download high resolution image

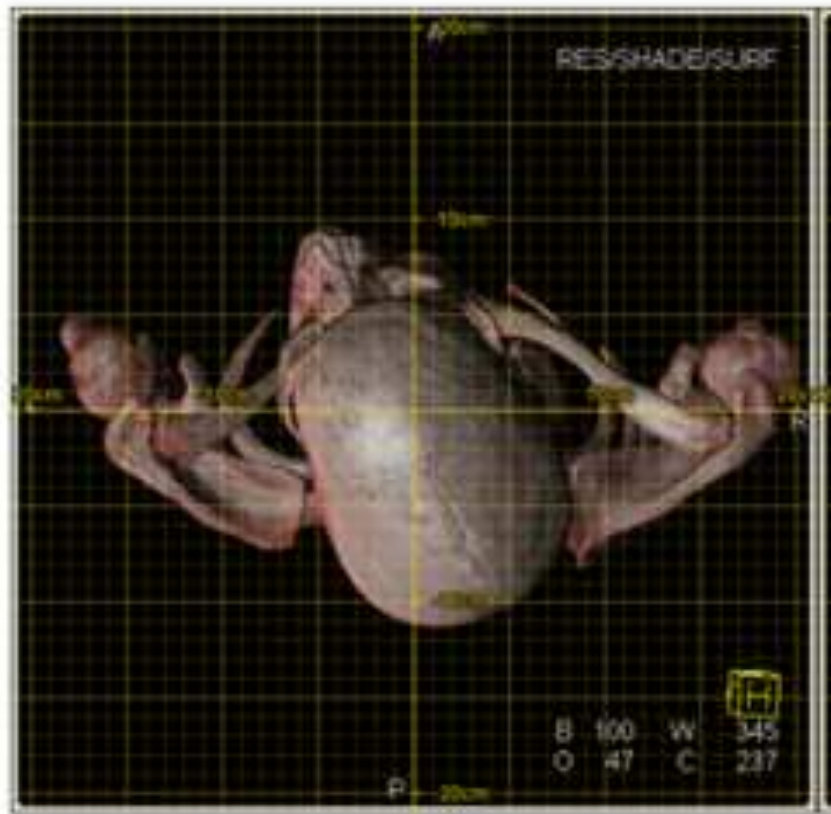

a.

b.

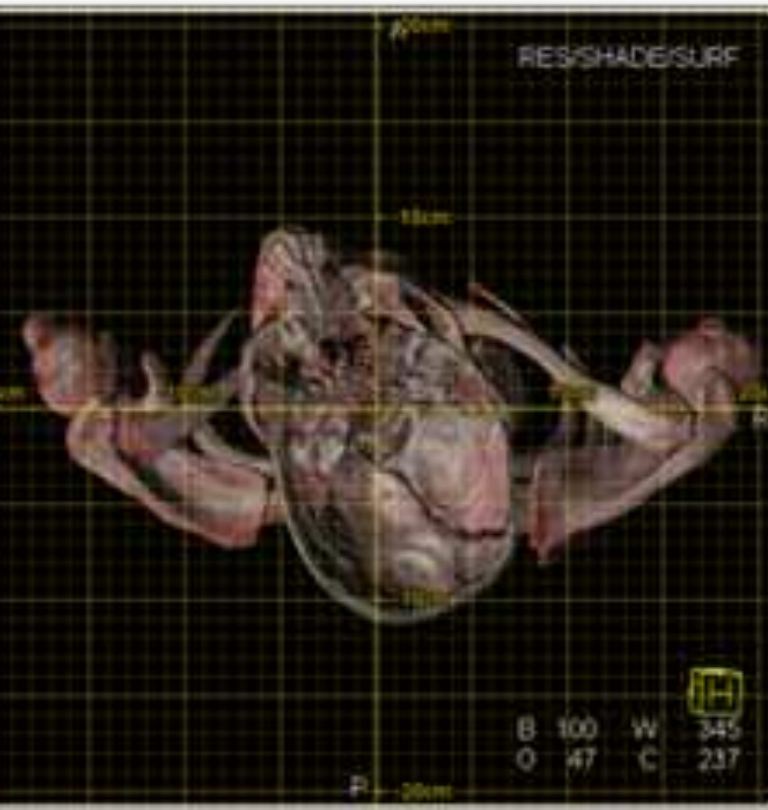

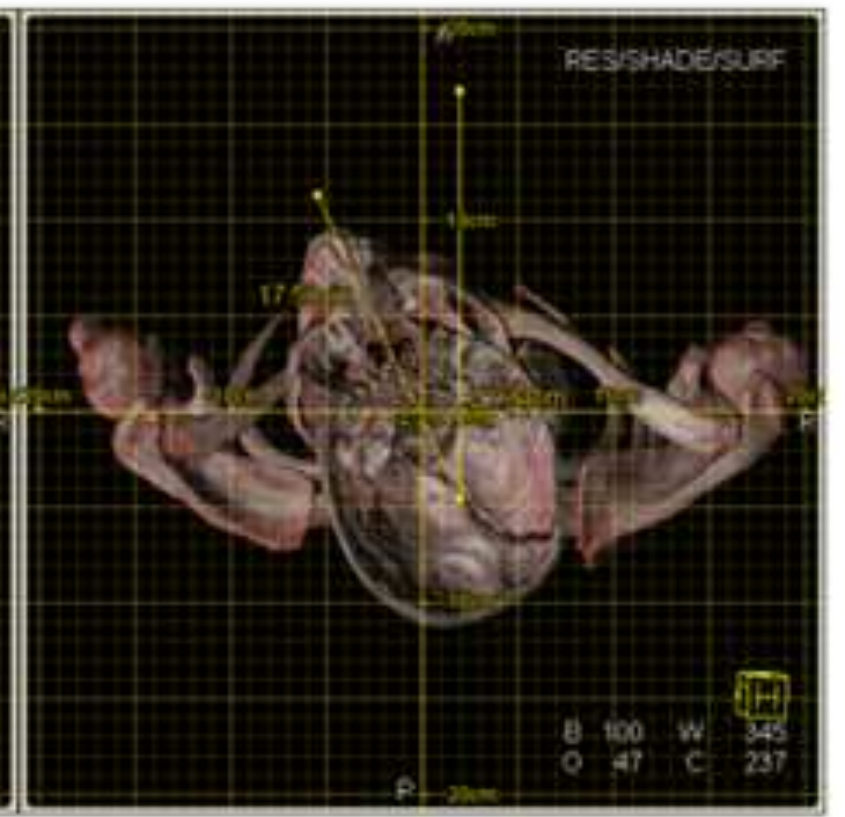

c. 


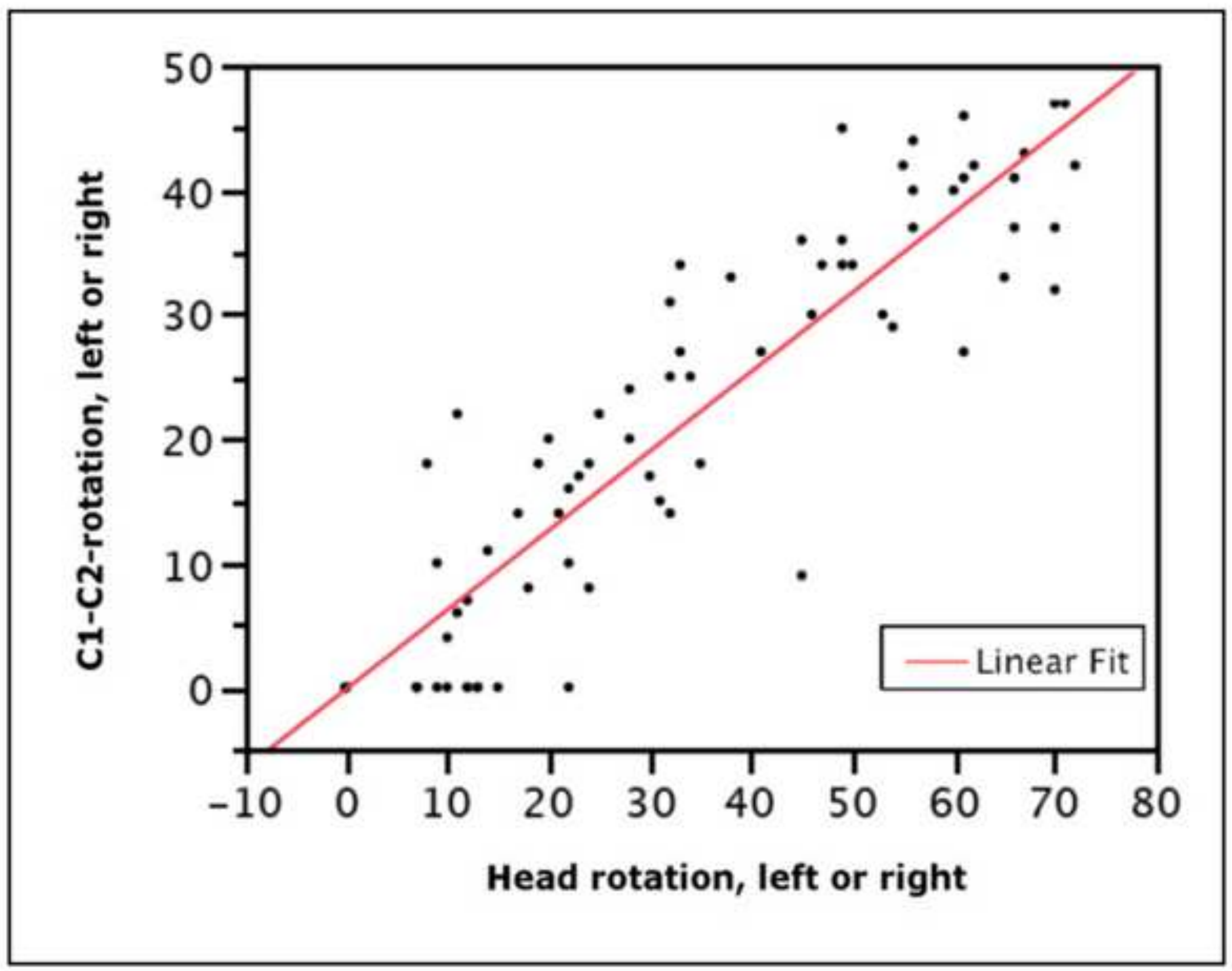

Figure 


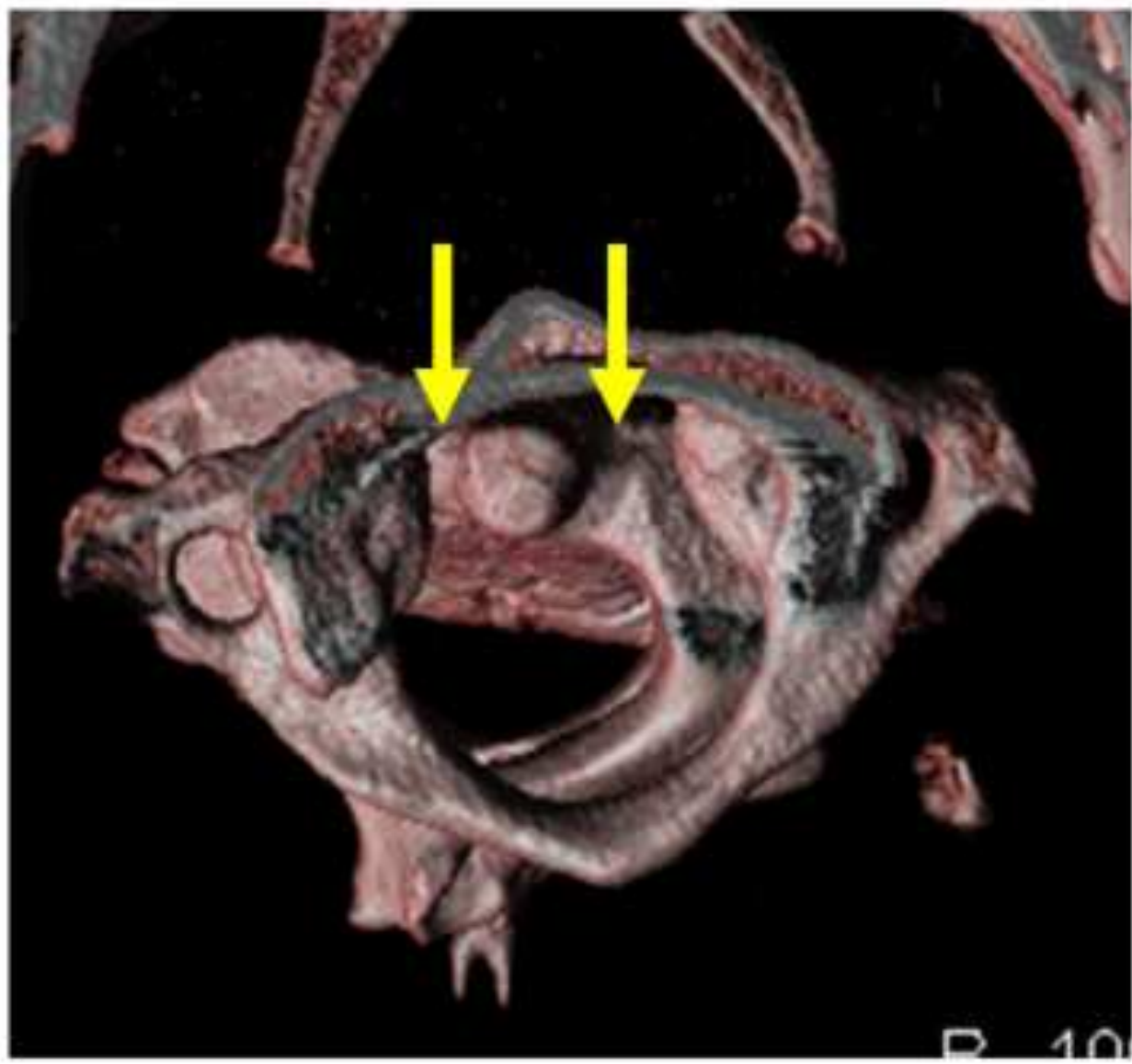

a.

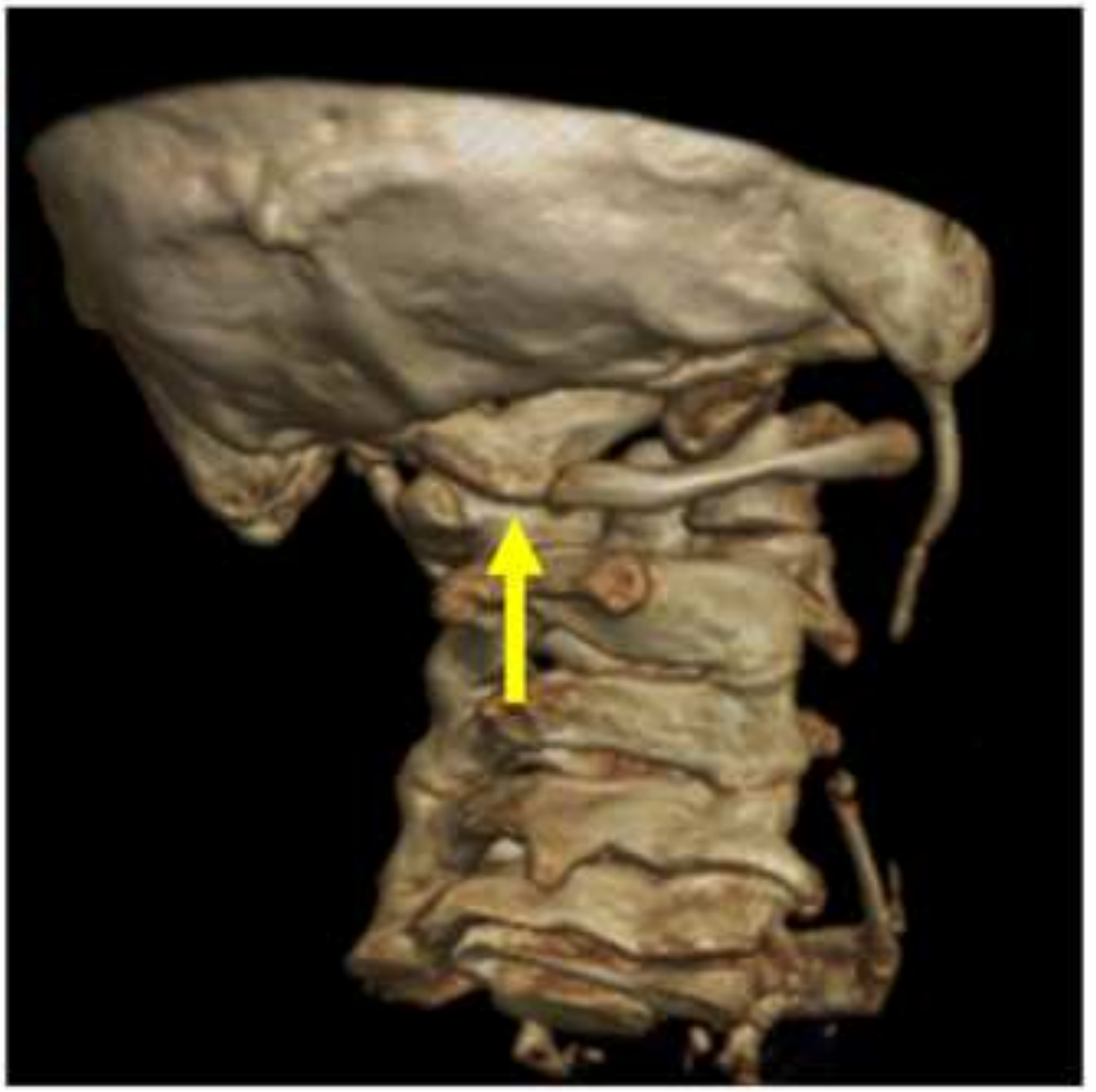

b.

Figure 
Table 1.

Head rotation, sex and stability of the cervical spine

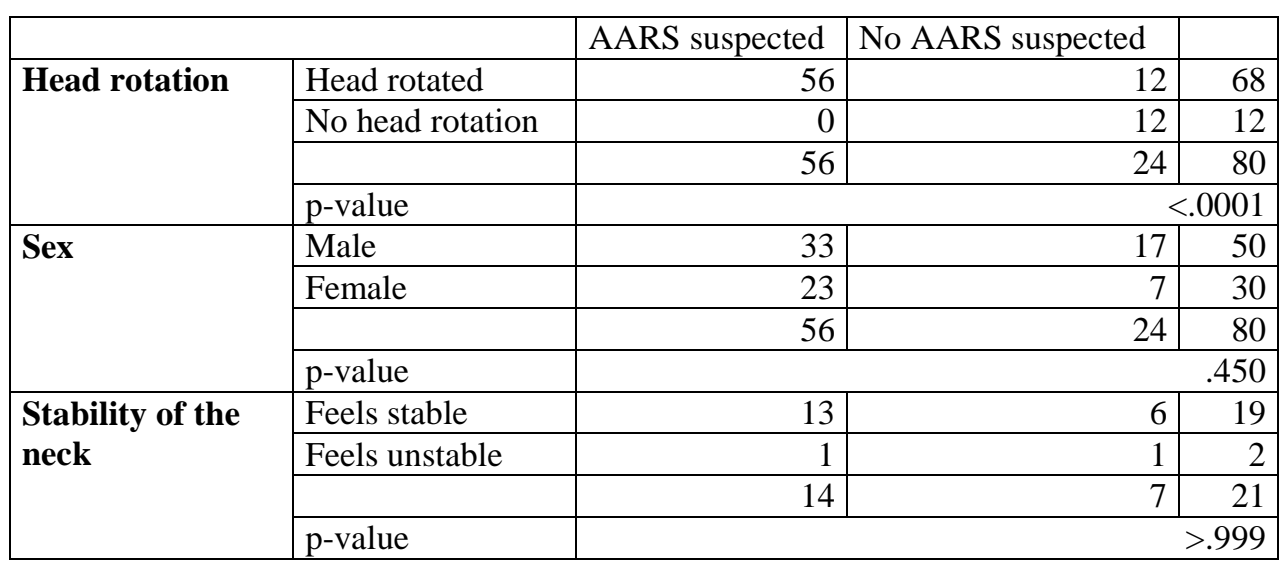


Table 2.

Continous data analysis

\begin{tabular}{|l|r|r|r|r|r|r|r|}
\hline & $\mathrm{N}$ & $\min$ & $\max$ & median & mean & SD & p-value \\
\hline Age (years) & 80 & .04 & 87 & 40.5 & 37.1 & 24.8 & .378 \\
\hline Lenght $(\mathrm{cm})$ & 77 & 51.0 & 191 & 168 & 151 & 40.1 & .765 \\
\hline Weight $(\mathrm{kg})$ & 77 & 2.30 & 114 & 62.0 & 55.8 & 28.1 & .239 \\
\hline BMI (kg/m ${ }^{2}$ ) & 77 & 6.84 & 36.8 & 21.4 & 21.3 & 5.50 & .209 \\
\hline Ethyl alcohol in blood (ppt) & 70 & 0 & 3.7 & 0 & .72 & 1.1 & .905 \\
\hline Ethyl alcohol in urine (ppt) & 62 & 0 & 5.0 & 0 & .97 & 1.3 & .662 \\
\hline Certain time from death to CMIV (h) & 32 & 9 & 110 & 66.5 & 65.8 & 31.3 & .053 \\
\hline Approximated time from death to CMIV (h) & 48 & 9 & 235 & 54.5 & 69.0 & 46.1 & .657 \\
\hline Time (certain+app.) from death to CMIV (h) & 80 & 9 & 235 & 57.0 & 67.7 & 40.6 & .334 \\
\hline
\end{tabular}


Table 3.

Cause of death

\begin{tabular}{|l|r|r|r|r|}
\hline & $\mathrm{N}$ & AARS suspected & No AARS suspected & p-value \\
\hline Fatal hemorrhage & 36 & 24 & 12 & .628 \\
\hline Neurologic/head-/neck injury & 13 & 9 & 4 & $>.999$ \\
\hline CO-poisoning/fire/heat stroke/burn & 4 & 2 & 2 & .579 \\
\hline SIDS & 6 & 3 & 3 & .358 \\
\hline Lung injury & 8 & 7 & 1 & .424 \\
\hline Asphyxia/strangulation & 8 & 7 & 1 & .424 \\
\hline Drowning & 3 & 2 & 1 & $>.999$ \\
\hline Heart injury & 5 & 2 & 3 & .156 \\
\hline Poisoning/drugs & 4 & 3 & 1 & $>.999$ \\
\hline Unknown & 4 & 4 & 0 & .311 \\
\hline Total number of cases & $\mathbf{8 0}$ & $\mathbf{5 6}$ & $\mathbf{2 4}$ & \\
\hline
\end{tabular}


Table 4.

Autopsy results on head and neck

\begin{tabular}{|c|c|c|c|c|}
\hline & $\mathrm{N}$ & AARS suspected & No AARS suspected & P-value \\
\hline Injuries/pathologic changes of the head & 45 & 32 & 13 & .811 \\
\hline Injuries/pathologic changes of the neck & 34 & 26 & 8 & .330 \\
\hline Fracture $\mathrm{C} 0-\mathrm{C} 1-\mathrm{C} 2$ & 5 & 4 & 1 & $>.999$ \\
\hline Deep soft tissue injury, level C0-C1-C2* & 4 & 3 & 1 & $>.999$ \\
\hline Fracture C3-C7 & 2 & 2 & 0 & $>.999$ \\
\hline Deep soft tissue injury, level C3-C7* & 4 & 3 & 1 & $>.999$ \\
\hline Total number of cases & 80 & 56 & 24 & \\
\hline
\end{tabular}

